\title{
Correction to: Identification of tRNA nucleoside modification genes critical for stress response and development in rice and Arabidopsis
}

Youmei Wang ${ }^{1,2}$, Chaoqun Pang ${ }^{1,2}$, Xukai $\mathrm{Li}^{5}$, Zhen $\mathrm{Hu}^{1,2}$, Zhengyi $\mathrm{Lv}^{1,2}$, Bo Zheng ${ }^{3,4}$ and Peng Chen ${ }^{1,2^{*}}$

\section{Correction}

Following publication of the original article [1], the authors reported that there was a mistake in the presentation of their funding information. The sentence "This study was supported by the National Natural Science Foundation of China (31,100,268 to Peng Chen, 31,270,658 to Bo Zheng);" should instead read "This study was supported by the National Natural Science Foundation of China (31100268 to Peng Chen, 31370604 to Bo Zheng);".

\footnotetext{
Author details

${ }^{1}$ Biomass and Bioenergy Research Centre, Huazhong Agricultural University, Wuhan 430070, China. ${ }^{2}$ College of Plant Science and Technology, Huazhong Agricultural University, Wuhan 430070, China. ${ }^{3}$ Key Laboratory of Horticultural Plant Biology of Ministry of Education, Huazhong Agricultural University, Wuhan 430070, China. ${ }^{4}$ College of Horticulture and Forestry Sciences, Huazhong Agricultural University, Wuhan 430070, China. ${ }^{5}$ College of Life Sciences, Shanxi Agricultural University, Taigu, Shanxi Province 030801, China.
}

Received: 29 January 2018 Accepted: 29 January 2018

Published online: 19 February 2018

\section{Reference}

1. Wang Y, Pang $C, L i X, H u Z$ Z, Lv Z, Zheng B, Chen P. Identification of tRNA nucleoside modification genes critical for stress response and development in rice and Arabidopsis. BMC Plant Biol. 2017;17:261. https://doi.org/10.1186/ s12870-017-1206-0.

\footnotetext{
* Correspondence: chenpeng@mail.hzau.edu.cn

${ }^{1}$ Biomass and Bioenergy Research Centre, Huazhong Agricultural University, Wuhan 430070, China

${ }^{2}$ College of Plant Science and Technology, Huazhong Agricultural University, Wuhan 430070, China
} 